\title{
Ordre et désordre : le Montreal Lunatic Asylum et la naissance de l'asile au Québec
}

\section{André Cellard et Dominique Nadon}

Volume 39, numéro 3, hiver 1986

URI : https://id.erudit.org/iderudit/304371ar

DOI : https://doi.org/10.7202/304371ar

Aller au sommaire du numéro

Éditeur(s)

Institut d'histoire de l'Amérique française

ISSN

0035-2357 (imprimé)

1492-1383 (numérique)

Découvrir la revue

Citer cet article

Cellard, A. \& Nadon, D. (1986). Ordre et désordre : le Montreal Lunatic Asylum et la naissance de l'asile au Québec. Revue d'histoire de l'Amérique française, 39(3), 345-367. https://doi.org/10.7202/304371ar d'utilisation que vous pouvez consulter en ligne. 


\title{
ORDRE ET DÉSORDRE: LE MONTREAL LUNATIC ASYLUM ET LA NAISSANCE DE L'ASILE AU QUÉBEC ${ }^{1}$
}

\author{
ANDRÉ CELLARD \\ Département d'histoire \\ Université d'Ottawa \\ DOMINIQUE NADON \\ École de médecine \\ Université d'Ottawa
}

\begin{abstract}
«...there are some points brought under my notice by Sir John Dorrat, to which I think it is important that the attention of Your Majesty's Government should be directed without delay. I advert to the want of any public establishment for the reception of insane persons either in Lower or Upper Canada.» (Lord Durham) ${ }^{2}$
\end{abstract}

Il est généralement reconnu que l'asile de Beauport, qui ouvrit ses portes en 1845, fut le premier asile au Canada français et qu'il s'agissait de la première institution du genre au Québec ${ }^{3}$. Dans leur ouvrage Essais pour une préhistoire de la psychiatrie au Canada (1800-1885), André Paradis et ses collaborateurs font néanmoins état, de façon très brève, de l'existence du Montreal Lunatic Asylum puisqu'ils ont trouvé quelques maigres documents à son sujet: un énoncé des règlements de l'institution, une loi accordant des crédits à l'asile et un rapport d'une

1 Ce texte est le résultat de la mise en commun de deux recherches. Dominique Nadon s'est d'abord intéressé aux aspects institutionnels et thérapeutiques du Montreal Lunatic Asylum. André Cellard, quant à lui, s'est surtout penché sur l'étude des divers facteurs sociaux, politiques et économiques qui conduisirent à la fondation de cet asile et à la place qu'il occupe dans l'évolution de la psychiatrie asilaire au Québec. Nous tenons à remercier MM. Toby Gelfand et André LaRose, du département d'histoire de l'Université d'Ottawa. Les nombreux conseils qu'ils nous ont prodigués nous ont été vraiment utiles. Nos remerciements vont aussi à l'Institut Hannah, pour l'histoire de la médecine, pour l'aide financière qu'il a généreusement accordée aux deux signataires de cet article.

(John George Lambton), Earl of Durham, «Report on the Affairs of British North America...», dans Irish University Press of British Parliament Papers, Colonies, Canada, vol. 2, Session 1839 (Shannon, 1968), 49.

3 Voir H. Hurd, The Institutional Care of the Insane in the United States and Canada (Baltimore, John Hopkins University Press, 1916), 129-132; M. E. Abott, History of Medicine in the Province of Quebec (Montreal, McGill-Queen's University Press, 1931), 77-79; V. E. Appleton, «Psychiatry in Canada a Century ago», CPCJ, 12 (août 1967): 345-361; A. Paradis et al., Essais pour une préhistoire de la psychiatrie au Canada (1800-1885), suivi d' une anthologie de textes, (Université du Québec à Trois-Rivières, département de philosophie, 1977), coll. «Recherches et Théories», no 15; et plus récemment, F. Boudreau, De l'asile à la santé mentale (Montréal, Éditions Saint-Martin, 1984) 21-33 
page, imprimé dans la Montreal Medical Gazette de 1844. Étant donné le peu de renseignements dont ils disposaient, les auteurs ont qualifié l'institution d'énigmatique, se bornant à dire à ce propos:

En effet, bien que partout et toujours Beauport soit présenté comme le premier asile ayant existé au Bas-Canada, nous avons la preuve qu'avant 1845 un autre asile avait pignon sur rue à Montréal (...) Le silence total observé au sujet de cet asile nous fonde à croire qu'il s'agissait d'une institution à caractère d'abord privé, d'une part. D'autre part, comme le gouvernement lui octroie une subvention, au moinș une fois, nous pouvons en déduire qu'il hébergeait probablement certains cas que l'assistance publique lui envoyait. ${ }^{4}$

Les Archives publiques du Canada renferment pourtant un grand nombre de documents permettant de mieux connaitre cette institution qui a jusqu'ici paru mystérieuse. Il est donc possible d'affirmer que le Montreal Lunatic Asylum fut le premier asile établi au Québec. Destiné à la garde et au soin des aliénés, cet asile était public et entièrement subventionné par l'État. Il ouvrit ses portes le premier novembre 1839 et, à l'instar d'autres asiles de l'époque, avait été aménagé dans l'édifice même de la prison commune de Montréal ${ }^{5}$. Aux yeux des autorités, il s'agissait-là d'une solution provisoire, en attendant la construction d'un asile d'une centaine de lits en périphérie de la ville. Toutefois, ce second projet n'eut pas de suite; s'il avait abouti, le premier asile permanent au Québec aurait été public et situé à Montréal et non à Beauport ${ }^{6}$.

L'étude du Montreal Lunatic Asylum est d'autant plus intéressante qu'elle constitue plus que l'histoire d'une institution en particulier; elle permet en définitive de voir sous un jour tout à fait nouveau les divers mécanismes qui marquèrent au Québec la prise de gestion directe du traitement des aliénés par les autorités civiles. A vrai dire, l'apparition, en 1839, d'un asile au Bas-Canada n'avait rien de fortuit et ce n'est certes pas un hasard non plus si c'est pratiquement en même temps que les autorités gouvernementales du Haut-Canada décidaient elles aussi de s'équiper d'institutions de ce genre? ${ }^{7}$. Nous croyons que c'est suite

\footnotetext{
4 Paradis et al., Essais pour une préhistoire..., 162.

A cette époque, l'asile de Toronto, par exemple, était situé dans l'ancienne prison de la ville de York (1841-1846). Voir Hurd, The Institutional Care..., 129-132, et D. Francis, «The Development of the Lunatic Asylum in the Maritime Provinces» in S. E. D. Short, ed., Medicine in Canadian Society - Historical Perspectives (Montreal, McGill-Queen's University Press, 1981), 93-114. La «Montreal Geol» ou prison «Au pied du Courant», quant à elle, fut le site de l'exécution de 12 patriotes en 1838. Elle avait été bâtie quelques temps auparavant et complétée vers 1838 , alors qu'elle servit à l'incarcération de plusieurs centaines de prisonniers politiques de l'insurrection de 1837-38.

6 Archives publiques du Canada (ci-après APC), Fonds Lunatic Asylum, RG 4 B 65, vol. 2, dossier 1843-44.

7 T. Brown, «The Origins of the Asylum in Upper Canada, 1830-1839», Bulletin canadien d'histoire de la médecine, 1,1: 27-59.
} 
aux bouleversements engendrés par la crise économique et par les pressions démographiques qui menèrent aux Rébellions de 1837-38 que naquit l'institution asilaire au Bas-Canada. Les Britanniques imposèrent en quelque sorte l'asile aux Bas-Canadiens - qui jusque-là faisaient figure de retardataires en ce qui concerne le traitement des maladies mentales - parce qu'ils voyaient dans l'implantation d'une telle institution l'une des solutions aux causes des désordres récents. L'histoire de l'asile, que ce soit ici, en Europe ou dans le reste de l'Amérique du Nord, est donc indissociablement liée à divers facteurs économiques et sociaux qui en favorisèrent l'apparition. Il convient donc, avant de retracer la brève histoire du Montreal Lunatic Asylum, d'effectuer un survol des principaux courants qui entraînèrent la naissance de l'asile en Occident et au Québec. Cela devrait nous permettre de mieux comprendre les mécanismes internes et externes qui régirent, au cours de ses six années d'activité, la vie de la première institution asilaire bascanadienne et qui finalement conduisirent à sa fermeture en 1845 lors de la fondation de l'asile de Beauport.

\section{1 - L'AVENEMENT DE L'ASILE}

\section{a - L'Occident et le Québec}

Les premières mesures importantes à l'égard des malades mentaux furent prises en Occident au cours de la seconde moitié du 17e siècle, alors que les monarchies - de plus en plus centralisatrices - entreprirent, avec l'appui de la bourgeoisie naissante, d'enfermer les fauteurs de troubles, les déviants et les improductifs qui peuplaient leurs royaumes. C'est ce que Foucault a appelé «le grand renfermement», renfermement qui allait se concrétiser en France par l'ouverture d'hôpitaux généraux et en Angleterre par la fondation de workhouses et d'almhouses ${ }^{8}$. Enfermés avec les vagabonds, les prostituées et les criminels, les malades, les infirmes et les orphelins, les «fous» quittèrent alors un univers flou et indéfini pour accéder à celui de la criminalité et de l'indigence. Jusqu'à la fin du $18 \mathrm{e}$ siècle, le traitement des «insensés» allait ainsi relever de l'incarcération plutôt que de la thérapeutique. A cette époque cependant, l'Anglais Samuel Tuke et le Français Philippe Pinel «libérèrent» les malades mentaux en inventant, chacun à leur façon, l'asile et le traitement moral, qui devinrent rapidement la nouvelle panacée occidentale contre la folie. L'asile était une institution complètement réservée aux aliénés, qui y étaient «soignés» sans cachots ni chaînes, par le traitement moral, une sorte de thérapie des moeurs qui avait pour but de permettre aux patients de se comporter à nouveau selon la norme. C'est ainsi qu'entre 1815 et 1830, l'Angleterre s'équipa d'un réseau national d'asiles tandis que plus près de nous, les Américains attendirent les années 1830 avant de faire de même.

8 M. Foucault, Histoire de la folie à l'âge classique (Paris, Plon, 1960). 
L'apparition de l'asile au tournant du 19e siècle n'est cependant pas accidentelle; elle ne résulte pas non plus uniquement de la générosité désintéressée des «philanthropes» de l'époque. En effet, la naissance de l'asile en Occident est liée à l'accroissement de l'indigence urbaine engendrée par l'industrialisation et la transition au capitalisme. Effrayés par les masses ouvrières venues s'entasser dans les villes et par le nombre toujours croissant de défavorisés que laissait derrière elle chaque fluctuation du nouvel ordre économique, les mieux nantis entreprirent d'ordonner et de catégoriser le monde de la misère urbaine, afin d'exercer, sur les «classes dangereuses», un contrôle plus efficace. C'est ainsi qu'en même temps que l'asile furent mis sur pied les pénitenciers, les maisons de redressement et autres institutions dites «de charité», qui avaient pour fonction d'isoler les différentes catégories de déviants - source de désordre et d'inquiétude - dans le but de faire de ces individus des êtres moraux et productifs.

En comparaison avec les services déjà disponibles dans la plupart des pays occidentaux, le Québec de la première moitié du $19 \mathrm{e}$ siècle réservait quant à lui un sort bien peu enviable à ses malades mentaux, en matière de soins et d'installations. En effet, la situation des aliénés canadiens n'avait pratiquement pas évolué depuis 1717, époque à laquelle quelques loges - sortes de cachots individuels - avaient été aménagées dans l'enceinte de l'Hôpital général de Québec.

Certes, on avait discuté dès 1793 à la Chambre d'assemblée du Bas-Canada d'un «Acte pour soulager les familles du soin de pourvoir pour les foux ( $\mathrm{sic}$ ) et les gens en démence» ${ }^{9}$ mais, il faut tout de suite préciser que cette loi n'entretenait aucun rapport avec les réformes de Pinel et de Tuke et les quelques cellules de l'édifice qui fut construit à cette époque près de l'Hôpital général de Montréal ne servirent qu'à l'internement d'un petit nombre de malades, détenus sans aucun traitement; en 1830, du reste, on n'y comptait plus que deux insensées, enfermées depuis 1793 et 1796 respectivement ${ }^{10}$. Malgré la décision, en 1801, du Conseil législatif de venir en aide financièrement aux communautés religieuses pour la garde des insensés et des enfants abandonnés et la nomination, par la suite, de commissaires chargés de la supervision de ce programme, les efforts des pouvoirs politiques de l'époque n'allaient servir, en définitive, qu'à perpétuer ici un mode de traitement d'Ancien Régime ${ }^{11}$.

En 1823, par suite d'une dispute en Chambre d'assemblée concernant le montant des subsides accordés à l'entretien des aliénés, le Con-

\footnotetext{
$9 \quad$ La Gazette de Montréal, 21 mars 1793.

10 Sir John Doratt, «Observations... on the Custody of the Insane, and the Expediancy of a Public Asylum», Appendice A du rapport Durham, op. cit., 9-10.

11 Sur cette question, voir A. Paradis et al., «L'émergence de l'asile québécois au XIXe siècle», Santé mentale, 2,2 (nov. 1977): 1-8.
} 
seil Législatif décida de faire enquête sur leur situation et sur celle des enfants abandonnés au Bas-Canada. En 1824, le comité nommé à cette fin critiqua fortement dans son rapport le système des loges destiné à la garde des insensés ${ }^{12}$. On déplorait surtout «qu'on ait employé dans les différents Districts, pour ces misérables expédiens (sic) (l'internement dans les loges), des sommes si considérables qu'elles auraient suffi à former un asile pour les Lunatiques ...» ${ }^{13}$. Le rapport recommandait vivement la construction d'un asile d'une soixantaine de places au moins, «afin de combiner l'utilité générale avec l'économie...» ${ }^{14}$. Cette solution avait en effet l'avantage, selon les auteurs du rapport, de s'avérer, à moyen terme, beaucoup moins coûteuse que le systéme des loges. Le rapport resta toutefois lettre morte.

En fait, les conditions de détention des aliénés au Bas-Canada eurent plutôt tendance à se détériorer par la suite. A partir de 1831, par exemple, les religieuses de l'Hôpital général de Montréal refusèrent de prendre soin des malades mentaux de la région; ceux qui ne pouvaient être gardés à la maison se retrouvaient donc enfermés à la prison commune ${ }^{15}$. Dans les villes de Trois-Rivières et de Québec par ailleurs, il n'y avait qu'une vingtaine de places tout au plus qui étaient spécifiquement destinées à la garde des insensés dans les hôpitaux locaux. Il semble cependant que le sort des «bénéficiaires» qui en faisaient usage n'était en rien enviable à celui des malades qui devaient se contenter de la prison. A titre d'exemple, on pourrait citer les conditions misérables dans lesquelles étaient entretenus ces patients à l'Hôpital général de Québec. A l'intérieur de minuscules cellules - la plupart d'entre elles mesuraient huit pieds de long sur sept pieds six pouces de large et huit pieds de haut - le malade se retrouvait dans un isolement complet. Quelquefois enchaîné, il ne sortait de son réduit qu'une fois tous les huit jours pour être conduit dans une autre cellule tandis qu'on nettoyait la sienne ${ }^{16}$. En 1845, le Grand Jury de la ville et du district de Québec s'indignait après une visite effectuée aux loges de cette institution:

12 «Rapport du Comité Spécial nommé pour s'enquérir et faire rapport sur les établissements de cette Province, pour la réception et la guérison des Personnes dérangées dans leur esprit, pour la réception des enfants trouvés, et pour le soulagement et la guérison des Personnes malades et infirmes, avec les Dépenses d'iceux défrayés à mểme le Revenu Provincial etc..., Journal du Conseil Législatif, 1824, Appendice 1.

13 «Rapport du Comité Spécial... Appendice A (no 1). Il est à noter cependant que le Rapport fait mention à quelques reprises de six «cellules morales» à l'Hôpital général de Québec. Destinées, semble-t-il, au soin thérapeutique des aliénés qui pouvaient présenter quelque espoir de guérison, elles avaient été bâties vers 1818. C'est le Dr Hackett, médecin de l'institution, qui avait été le principal promoteur de cette initiative locale. Voir «Rapport du Comité spécial...», Appendice D (no 3), ainsi que P. Keating, «L'émergence de l'asile au Québec (1800-1845)», communication présentée à la réunion annuelle de la Société Canadienne pour l'Histoire de la Médecine, Montréal, mai 1985.

14 «Rapport du Comité Spécial...», Appendice A (no 1).

15 Paradis, art. cit., 1-8; aussi, APC, RG $4 \mathrm{C1}$, vol. 8, doc. 1369, les commissaires à Murdoch, 31 mars 1840.

16 Hurd, The Institutional Care..., 252-255. 
The misery endured by its outcast and infortunate inmates is extreme. Their filthy cells are even in this hot season quite damp and unfit for the habitation of even animals much less of human beings who in addition to an afflicting visitation of providence are then left in a continually putrid atmosphere, and with hardly a ray of light of Heaven to pine away and suffer an unmerited punishment which an incarceration in their horrible dungeons certainly is. ${ }^{17}$

Parce que la prise en charge directe de la garde des aliénés à l'intérieur d'institutions spécifiquement destinées à cette fin n'avait pas encore été jugée nécessaire, ni par le gouvernement, ni par la population en général, le Bas-Canada accusait donc en cette première moitié du 19e siècle, un certain retard par rapport aux réformes européennes et américaines en ce qui a trait au traitement de la maladie mentale. Reste à savoir pourquoi.

\section{b - Les pressions économiques et sociales: ferment de l'asile}

Tel qu'indiqué précédemment, l'apparition de l'asile n'est pas le fruit d'une évolution scientifique rationnelle; en d'autres mots elle ne survint pas de façon automatique, suivant la «marche du progrès», comme il serait si facile de le croire. Elle correspond plutôt à l'évolution de certaines conditions socio-économiques qui rendirent l'avènement d'une telle institution désirable aux yeux de certains groupes sociaux. Encore à l'âge du mercantilisme, le Bas-Canada n'offrait pas, dans la première moitié du $19 \mathrm{e}$ siècle, les conditions socio-économiques propices à l'érection d'asiles. L'industrialisation n'avait pas encore eu prise dans cette société où près de $80 \%$ de la population habitait la campagne et où le réseau des solidarités familiales et villageoises arrivait encore à absorber les indigents. De plus, l'élite canadienne-française, principalement composée de membres des professions libérales pratiquant hors de la ville, avait, de façon générale, les yeux tournés surtout vers la campagne, là où au Bas-Canada s'étaient maintenues les structures traditionnelles ${ }^{18}$. Elle ne voyait donc pas la nécessité de construire des asiles - institutions essentiellement urbaines - et c'est sans doute pourquoi le rapport de 1824 n'eut aucune suite. En somme, rien à ce momentlà ne semblait inciter les Canadiens - les Canadiens français, en particulier - à la construction d'asiles «modernes» pour le soin des aliénés.

Les conditions sociales, économiques et politiques allaient toutefois évoluer au Bas-Canada en cette première moitié du 19e siècle et c'est une crise économique et démographique grave - celle-là même qui

\footnotetext{
${ }^{17}$ APC, RG 4 B 65, vol. 2, dossier 1843-44, le Grand Jury de la ville et du district de Québec à la Cour des Sessions de la paix du district de Québec, juillet 1844 .

18 Pour une analyse de la structure sociale bas-canadienne, voir F. Ouellet, Histoire économique et sociale du Bas-Canada (Montréal, Fides, 1966), ainsi que Le Bas-Canada, changements structuraux et crise (Ottawa, Éditions de l'Université d'Ottawa, 1976).
} 
mena aux Rébellions de 1837-38 - qui permit l'émergence des conditions sociales et psychologiques favorables à l'érection d'asiles ${ }^{19}$. En effet, ce n'est pas l'industrialisation, mais bien la crise agricole, qui privait les Canadiens français de terres et forçait l'exode d'une partie d'entre eux vers les villes, d'une part, et l'arrivée massive d'immigrants britanniques, d'autre part, qui allaient, de concert, faire croître la misère et l'indigence urbaines de façon spectaculaire. Or ces perturbations mirent fortement à l'épreuve le réseau d'institutions de «bienfaisance», déjà jugé insuffisant. Les désordres résultant de l'évolution de la conjoncture suscitèrent l'inquiétude de la bourgeoisie citadine, d'origine britannique en grande partie. Mais les Rébellions de 1837-38, en réduisant à néant l'influence de l'élite canadienne-francaise du parti Patriote, permirent à l'élite anglophone de réaliser certains de ses objectifs les plus pressants en matière de contrôle social, et ce, par le biais du gouvernement britannique qui assumait désormais directement le pouvoir politique au Bas-Canada.

En 1838, Lord Durham fut envoyé par Londres dans les Canadas afin de trouver des solutions aux problèmes qui s'y posaient. L'assimilation des Canadiens français constituait l'une des mesures proposées par Durham dans son fameux rapport. Selon ce gouverneur anglais, il suffirait pour y parvenir de noyer les Canadiens français sous le flot des immigrants britanniques qui débarquaient année après année au port de Québec. Or, comme les nouveaux arrivants ne trouvaient pas au BasCanada les structures et les institutions auxquelles ils étaient habitués dans la mère patrie, nombre d'entre eux partaient pour le Haut-Canada et de là, pour les États-Unis, déçus qu'ils étaient par la situation dans les colonies britanniques. Pour freiner cette saignée, Durham joignit sa voix à celle des pétitionnaires effrayés par les troubles récents ${ }^{20}$ et réclama, entre autres mesures, la construction d'asiles publics dans le Haut et le Bas-Canada ${ }^{21}$.

Bien souvent, en effet, ce sont, des préoccupations de la sorte généralement enrobées de considérations philanthropiques - que l'on retrouvait à la base des revendicatons de ceux qui désiraient au Canada la construction d'asiles. L'accroissement du nombre d'insensés démunis, conséquence de l'immigration massive des dernières années, et l'obligation pour plusieurs d'émigrer aux États-Unis afin d'y faire soigner les leurs incommodaient ces pétitionnaires, en très grande majorité issus de la classe supérieure anglophone. Ceux-ci ne pouvaient concevoir que le Bas-Canada, en dépit de son statut de colonie britannique,

\footnotetext{
19 Pour une étude des divers facteurs qui entraînèrent les Rébellions de 1837-38, voir $\mathrm{F}$. Ouellet, op . cit., et «Les insurrections de 1837-38: un phénomène social», dans Éléments d'histoire sociale au Bas-Canada (Montréal, Hurtubise HMH, 1972), 351-379.

20 APC, Archives du Secrétaire Provincial, Canada-Est, RG 4 C 1 et RG 4 B 65. Voir aussi Brown, «The Origins of the Asylum...»

21 Durham, Report on the Affairs..., 49
} 
soit privé d'un type d'institution commun à tout pays «civilisé»" ${ }^{22}$. On s'inquiétait aussi des désordres que pouvaient causer les insensés dans la population et à l'intérieur des prisons, comme en fait foi le texte d'une pétition datée du 16 février 1839:

That for a number of years past, the peace of the said dictrict (celui d'Ottawa) has been repeatedly disturbed and the moral feelings of its inhabitants shocked, by the appearances among them of maniacs, and insane persons, for the most part strangers to the country, or to the district. That although the magistrate have in every case, promptly, interfered both to protect the public, and to secure the unfortunate beings in question, yet their measures have been unavoidably attended with great public expense and inconvenience, owing to the necessity of confining and maintaining the deranged persons in the Common Gaol of the District.

...that it has become a solemn and imperious duty on the part of the Legislature to provide for the reception and accomodation of insane destitute persons of the Province, one large establishment worthy of the public character of Upper Canada, and of so just and philantropic a cause. ${ }^{23}$

Pour en revenir à Durham, il nous apparaît tout à fait cohérent d'ailleurs que l'érection d'asiles ait été suggérée dans un rapport destiné à trouver des remèdes aux désordres qui secouaient le Haut et le BasCanada, puisque l'ordre, la sécurité, la moralité et l'humanité représentaient - dans l'ordre - les principaux arguments des promoteurs de l'asile, du pénitencier, des maisons de correction et autres institutions «de charité». C'est en tout cas ce qui ressort du rapport de Sir John Dorrat, qui avait été mandaté par Durham afin de faire enquête sur les «institutions charitables» du Bas-Canada. Dans un plaidoyer en 13 points, intitulé «Observations (...) on the custody of the Insane, and the Expediency of a Public Asylum (in Lower Canada)», le commissaire, qui recommandait vivement la construction d'un asile public au Bas-Canada, allait jusqu'à faire le lien entre les Rébellions et la présence de lunatiques en liberté parmi les Canadiens français:

The great extent of forming the province of Lower Canada, and the number of lunatics dispersed over its surface without any prospect of relief, either temporarily or permanently, producing the worst influence over the moral character of society at large particularily in the more popular districts, inhabited principally by

22 APC, RG 4 B 65, vol 2. Il s'agit plus particulièrement de deux pétitions présentées au gouverneur général par des citoyens de la ville de Montréal en juillet 1842 et en décembre 1844 . Une étude de quelque 1100 noms contenus dans ces deux pétitions nous a révélé que plus de $95 \%$ d'entre eux étaient de consonnance anglophone. Par ailleurs, la quasi-totalité des individus dont nous avons pu retracer la profession - qu'ils soient hommes d'affaires, marchands, avocats, médecins ou autres - faisaient partie de l'élite de la société montréalaise de l'époque.

23 Cité dans R. Splane, Social Welfare in Ontario, 1791-1893 (Toronto, University of Toronto Press, 1965), 203-304. 
Canadians, require the most serious consideration of the Legislature. $^{24}$

\section{2 - LE MONTREAL LUNATIC ASYLUM (1839-1845)}

\section{a - Solution provisoire et projet d'établissement permanent}

Le Rapport Durham fut publié le 11 février 1839 et le nouveau gouverneur général des Canadas, sir John Colborne, et son Conseil spécial furent priés par Londres de tout mettre en oeuvre afin que certaines recommandations du rapport fussent appliquées dans les plus brefs délais. Les choses n'allaient pas traîner en ce qui concerne l'établissement d'un nouvel asile d'aliénés au Bas-Canada. En effet, le 28 avril 1839, un mois après avoir essuyé le refus des gouverneurs du Montreal General Hospital d'ériger un asile sur leur terrain ${ }^{25}$, Colborne - qui par le passé ne semble pas avoir été particulièrement intéressé par la question ${ }^{26}$ - accordait au Secrétaire de la province une somme de 2000 livres, puisée à même les fonds publics, pour la mise sur pied au Bas-Canada d'un asile temporaire destiné au soin des insensés, en attendant la construction d'un établissement permanent ${ }^{27}$. Deux semaines plus tard, le 11 mai, une loi similaire était promulguée dans le HautCanada $^{28}$.

Le 27 juin 1839, une commission était mandatée afin de mettre le projet à exécution ${ }^{29}$. Il est intéressant de noter que chaque groupe ou sous-groupe qui avait un intérêt particulier à la construction d'asiles y était représenté, soit qu'il visait l'ordre public, ou qu'il escomptait un profit immédiat en termes d'emplois ${ }^{30}$. Composée de neuf membres, la Commission comptait en effet quatre médecins-chirurgiens (R. Robertson. J. Stephenson, J. Crawford et J. B. C. Thresler), trois hommes d'affaires (E. Guy, W. Lunn et J. Redpath) ainsi que deux hommes de loi (le juge Dominique Mondelet, membre du Conseil Spécial, et John Boston, seigneur de St-James et de Thwaites et futur shérif de Montréal). Pendant ses six années d'existence, la Commission connut successivement deux présidents (R.Robertson et J. Boston) et deux secrétaires (J. Stephenson et J. B. C. Thresler); elle garda cependant le même

\footnotetext{
Doratt, «Observations... on the Custody...», 10.

25 APC, RG 4 A1, Série G, vol. 576, A. Sakel au Secrétaire provincial, cité par P. Keating, loc. cit.

Brown, «The Origins of the Asylum...». Bien que réclamée par plusieurs, la construction d'un asile ne semble pas avoir été une priorité pour Colborne à l'époque où il était lieutenantgouverneur du Haut-Canada (1829-1836).

27 Ordonnances Provinciales du Bas-Canada, 4e session du Conseil Spécial (1839), 557 559.

28 Brown, «The Origins of the Asylum...», 32

29 APC, British Records, Pre-Confederation, Liber 16, vol. III, C3926-C3927, folio 148.

30 Dans le même ordre d'idées, il est à noter que J. B. C. Thresler se verra accorder le poste de médecin au Montreal Lunatic Asylum, tandis que le notaire Étienne Guy sera chargé des diverses transactions entourant l'achat d'un terrain choisi pour l'érection de l'asile permanent. Voir APC, RG 4 B 65, vol. 2.
} 
trésorier (W. Lunn). Les deux membres francophones ne semblent pas avoir pris une part active aux décisions de la Commission; c'est du moins l'impression qui se dégage à l'examen des documents qui sont d'ailleurs tous rédigés en langue anglaise.

Le mandat des commissaires était double: concrétiser le projet d'un asile permanent au Bas-Canada et, dans l'intervalle, y établir un asile temporaire. Les commissaires se mirent rapidement à l'oeuvre, comme en témoigne un document signé par le secrétaire provincial, le 6 août 1839:

His Excellency perceived with great satisfaction the steps which have been taken by you $(\ldots)$ and he fully concurs with you in opinion that it would be desirable to purchase the lot recommended containing about thirty arpents situated on the Côte St-Antoine at a cost of $£ 1050$. H. E. therefore will approve the measures being immediately adopted to secure the property and obtaining plans for an asylum of the improved modern system containing from 80 to 100 patients and which may be added thereafter. H. E. sees no reason why the foundation should not be proceeded with as soon as the purchase has been completed, he has no doubts that the legislature of the Province will provide ample means for the completion of the building. ${ }^{31}$

Le terrain fut acheté peu après et les plans du nouvel asile furent dessinés. La ville de Montréal avait été choisie comme site du premier asile permanent au Bas-Canada probablement parce que c'est là où le besoin s'en faisait le plus sentir. En effet, c'est dans la région de Montréal où la crise économique eut les plus graves effets; c'est là où se produisirent la quasi-totalité des troubles de 1837-38, c'est aussi là où se concentraient au Bas-Canada les immigrants britanniques démunis ${ }^{32}$. Par ailleurs, comme nous l'avons déjà signalé, les religieuses de l'Hôpital Général de Montréal refusaient la garde des malades mentaux depuis 1831.

Notons que le choix d'un site approprié à la construction d'un asile ne devait pas être fait au hasard. Il fallait respecter certains critères, ce dont s'acquittèrent avec soin les commissaires. Dans l'opinion des réformateurs sociaux de l'époque, un asile devait être situé dans un endroit sain, c'est-à-dire à l'extérieur de la ville, loin des milieux «avilissants». Il devait s'agir d'un emplacement discret, propice à l'éloignement des aliénés de la vue des bien-portants. Et, par-dessus tout, il importait que l'endroit choisi puisse permettre aux malades de se livrer à certaines tâches manuelles. Le travail était en effet un des éléments

\footnotetext{
31 APC, RG 4 C 1, vol. 3, doc. 434, le Secrétaire de la province aux commissaires du Montreal Lunatic Asylum, 6 août 1839.

32 F. Ouellet, «Structure des occupations et ethnicité dans les villes de Québec et Montréal (1819-1844)», Éléments d'histoire sociale..., 172-202.
} 
primordiaux de la thérapie asilaire, qui visait la réinsertion de l'aliéné dans la société en tant qu'élément productif. En d'autres termes, il fallait en refaire un «membre utile pour la société» ${ }^{33}$. Les commissaires furent donc fiers d'annoncer qu'ils avaient trouvé un terrain fort convenable, situé à deux milles de Montréal, sur la côte Saint-Antoine, bien dissimulé derrière la forêt; en outre, le sol s'y prêtait à l'agriculture, ce qui, selon eux, était «d'une importance considérable» ${ }^{34}$. De l'édifice projeté, on ne sait que peu de choses, cependant; il devait pouvoir accueillir une centaine de malades et bien entendu, il avait été dessiné de facon à ce que 25 de ces patients dits «de la classe supérieure» n'aient pas à se mêler aux 75 autres ${ }^{35}$. On estimait à 7000 livres le coût de sa construction $^{36}$.

En attendant la réalisation de ce projet, on avait jugé important de retirer les lunatiques des prisons dans lesquelles ils avaient été confinés pour la plupart et de les réinstaller dans un asile temporaire. Faute de mieux, les commissaires avaient dû opter pour le troisième étage de la prison de Montréal, qui avait servi jusque-là à l'emprisonnement des débiteurs:

They (les commissaires) also made considerable exertions to procure a private house, that could be converted into a temporary asylum, but were unsuccessful: and finally they suggested to his Excellency that a certain part of the jail should be alloted to them for the present accomodation of the insane, who had hitherto been kept indiscriminately with the other prisoners confined therein, both his Excellency and the Commissioners were all aware of the unsuitableness of the Commom Jail for the care of the insane persons, both as well for the sake of the establishment itself, as for the insane, but being persuaded that a few months would enable them to erect the proposed building, they expected that the accomodation would be requisite for a very short period only». ${ }^{37}$

De temporaire, cet asile allait néanmoins devenir permanent, jusqu'à sa fermeture en 1845. En effet, peu après le départ de Colborne en 1839, on vit s'effriter la volonté politique d'établir au Bas-Canada un asile public et ce, malgré les efforts répétés de la Commission. En guise de protestation, les commissaires démissionnèrent en bloc le 5 avril $1841^{38}$, mais rien n'y fit; l'érection d'un asile permanent - qui

\footnotetext{
33 APC, RG 4 B 65, vol. 2, dossier 1843-44.

34 APC, RG 4 B 65, vol. 2, dossier 1843-44, les commissaires au gouverneur général, 25 septembre 1843 .

35 APC, RG 4 B 65, vol. 2, dossier 1843-44, J. B. C. Thresler au gouverneur général, 25 septembre 1843 .

${ }_{36}$ APC, RG 4 B 65, vol. 2, dossier 1843-44, J. Boston et J. B. C. Thresler au gouverneur général, 23 août 1843 .

37 APC, RG 4 B 65, vol. 2, dossier 1843-44, J. Boston et J. B. C. Thresler au gouverneur général, 4 août 1842 .

${ }^{38}$ APC, RG 4 C 1, vol. 39, doc. 173, les commissaires au gouverneur général, 5 avril 1841. Le gouverneur les convainquit de revenir sur leur décision.
} 
pourtant semblait pressante en 1839 - n'était plus une priorité pour le gouvernement qui allait finalement en venir à une solution de compromis quelques années plus tard, avec l'ouverture d'un établissement semiprivé à Beauport. A quoi attribuer la mise au rancart du projet initial? Il est difficile pour le moment d'en déterminer la cause exacte, mais on peut penser que les nombreux problèmes législatifs, les crises politiques et les fréquents changements de gouverneur général qui survinrent dans la première moitié des années quarante furent autant d'obstacles à la réalisation du projet. D'autre part, il n'est pas exclu qu'une fois les Rébellions terminées et l'ordre revenu, les nouveaux gouverneurs aient tout simplement estimé que la construction d'un asile public permanent n'était plus urgente, voire même nécessaire.

\section{b - Un asile à part entière}

Quoi qu'il en soit, c'est dans une prison que fut installé le premier asile au Bas-Canada. Avec ses criminels et ses prostituées remplissant les deux premiers étages, cette institution, vue de l'extérieur, n'était pas sans rappeler les Hôpitaux généraux d'autrefois. Cependant, elle en différait à maints égards, à commencer par la ferme volonté qu'avaient les réformateurs de l'époque de séparer les insensés des criminels. En effet, puisque la réforme des marginaux, des indigents et des destitués impliquait un traitement différentiel suivant le type de déviance, une certaine classification s'avérait nécessaire afin de pouvoir diriger les déviants vers les institutions appropriées - asiles, pénitenciers, écoles de réforme et autres - qui leur étaient destinées ${ }^{39}$. Cette évolution se traduisit, du point de vue pratique, par le sentiment que les deux catégories de déviants, l'insensé et le criminel, ne pouvaient plus cohabiter et qu'il leur fallait désormais des institutions distinctes. On avançait souvent à cet égard l'argument que la prison était néfaste aux malades et n'encourageait pas leur guérison:

Your Lordship will therefore see that little advantage could be expected from the present arrangement other than removing the insane from the common cell, where they had been indiscriminately handled among the prisoners, exposed to their scopts and gests, and often deprived of their ordinary meals by the rapacity of their unfeeling associates... ${ }^{40}$

39 Interrogés dans le cadre de l'enquête de 1824, les shérifs de Québec et de Montréal considéraient en effet que la présence d'insensés dans leurs prisons constituaient «un obstacle à la classification des autres prisonniers». «Rapport du Comité Spécial..., Appendice A (no 8). Sur toute cette question, voir aussi J.-M. Fecteau, «Régulation sociale et répression sociale de la déviance au Bas-Canada au tournant du 19e siècle (1791-1815)», Revue d'histoire de l'Amérique française, 38,4 (printemps 1985): 499-521.

${ }_{40}$ APC, RG 4 C 1, vol. 39, doc. 173, les commissaires au gouverneur général, 5 avril 1841. 
Plus nombreux encore étaient ceux qui estimaient néanmoins, comme l'a noté Foucault ${ }^{41}$, que les insensés devaient plutôt être placés à l'écart parce qu'ils dérangeaient les prisonniers ${ }^{42}$.

En principe, l'asile était destiné à accueillir les individus «dérangés dans leur esprit» ou incapables d'exercer un «contrôle sur leur raison» - on les désignait alors par les vocables passe-partout de «lunatiques», d'«aliénés», ou d' «insensés» - susceptibles de nuire à l'ordre ou à la moralité publique ou qui constituaient une source de danger pour euxmêmes ou pour autrui. Comme c'est la famille qui jusque-là avait toujours été responsable de la garde des insensés, l'asile visait, au début surtout, les malades qui, dans le cadre urbain, étaient pour une raison ou pour une autre privés de foyer ou dont la famille n'avait pas les moyens de prendre $\operatorname{soin}^{43}$. On comprendra alors pourquoi la construction d'asiles s'avérait toujours plus impérieuse à mesure que s'accroissait la misère urbaine au Bas-Canada en cette première moitié du $19 \mathrm{e}$ siècle.

Ce sont les commissaires qui étaient responsables de l'admission des patients au Montreal Lunatic Asylum ${ }^{44}$. Le fait qu'une bonne partie des patients passèrent, au début en particulier, par la prison avant d'être admis dans un asile qui lui-même était installé à l'intérieur d'un édifice à caractère carcéral, ne doit pas encore une fois nous induire en erreur à propos de la spécificité du Montreal Lunatic Asylum. L'administration de cet asile était complètement indépendante de celle de la prison de Montréal et c'est pourquoi, par exemple, le Dr Arnoldi, médecin attitré de ladite prison, dut, à quelques reprises, se plaindre au Secrétaire général parce que les commissaires avaient refusé certains détenus qu'il avait tenté d'y faire admettre ${ }^{45}$. Les patients admis au Montreal Lunatic Asylum ne provenaient cependant pas tous des prisons locales. Leur nom pouvait être suggéré aux commissaires par un shérif, un médecin, un membre du clergé ou plus souvent encore par de simples particuliers $^{46}$. On avait prévu à cet effet un formulaire standardisé des-

\footnotetext{
41 Foucault, Histoire de la folie..., 401-439.

42 APC, RG 4 C 1, vol. 4, doc. 821, D. Arnoldi au gouverneur général, 24 février 1840 Voir aussi vol. 230, doc 1888 et vol. 224, doc. 1449.

43 Ce qui n'empêchait évidemment pas les gens aisés de vouloir faire soigner leurs insensés ou tout simplement de chercher à disposer de ceux qui pouvaient s'avérer dangereux. Comme on l'a vu plus tôt, ceux-ci disposaient généralement d'espaces réservés à l'intérieur de l'asile et les propriétaires de ces établissements comptaient beaucoup sur ces clients payants afin d'équilibrer leur budget. Voir «Rapport du Comité Spécial...», ainsi que A. Scull, Museums of Madness: The Social Organization of Insanity in Nineteenth Century England (New York, St.Martin's Press, 1979), 50-54 et «Rapport du Comité Spécial...», Appendice A (no 1).

44 Rules and Regulations Made and Established by the Chairman and the Commissioners of the Montrel Lunatic Asylum, for the Government of the Officers, Patients and Servants of the Institution (Montreal, James Starke and Co., 1840), 3.

45 Par exemple, APC, RG 4 C 1, vol. 4, doc. 821, D. Arnoldi au gouverneur général, 24 février 1840 .

46 Voir par exemple, RG 4 B 65, vol. 2, dossier 1845, Célestin Déry au Secrétaire provin-
} cial, 23 décembre 1845 . 
tiné à être rempli par le curateur ou par la personne légalement responsable du malade ${ }^{47}$.

Si l'asile constituait un instrument de régulation sociale visant à retirer de la circulation une certaine catégorie d'éléments perturbateurs, il n'en demeure pas moins qu'il avait aussi pour fonction la réforme ou la guérison des individus qui y étaient internés. C'est pourquoi, étant donné que les lieux étaient loin de correspondre à ce qui avait été prévu, des aménagements avaient été effectués au troisième étage de la prison afin que cet endroit soit en mesure de recevoir et de traiter les aliénés. Physiquement, l'asile était divisé en deux parties d'égale superficie, l'une pour les hommes, l'autre pour les femmes. Chaque section comptait dix pièces: huit d'entre elles (de 9 pieds sur 12 chacune) servaient de chambres pour les patients; une autre de chaque côté servait de salle de toilettes et une autre encore, dans le quartier des femmes, servait de cuisine et de salle de lavage. Dans la section des hommes, l'une des chambres servait à loger des gardiens. De plus, d'anciens passages avaient été modifiés afin d'y installer les appartements du surintendant et de la matrone (mari et femme). Chaque section était plus longue que large et elle était traversée par un corridor donnant sur un petit hall. Chacune était sous la surveillance d'un gardien du même sexe que les malades, employés qui s'ajoutaient au surintendant et à la matrone. Fait à noter, cette dernière gardait ses enfants avec elle à l'asile $^{48}$. La libre circulation des malades était d'usage, de même que la vie de groupe à la cuisine. Il est aussi fait mention par un serviteur $\mathrm{du}$ «salon des lunatiques» ${ }^{49}$, de salles de séjour (day-rooms) ainsi que de réfectoires ${ }^{50}$. Il se peut qu'il s'agisse-là de chambres ou de halls transformés à cet effet, à moins qu'ils n'aient été situés dans une autre aile du troisième étage.

Dès 1839, les patients du Montreal Lunatic Asylum reçurent les soins d'un médecin, soins qui devinrent réguliers à partir de 1841 alors que le docteur Thresler (qui était aussi l'un des membres de la Com-

\footnotetext{
47 Rules and regulations..., 15: «Gentlemen, Upon your admitting A. B. into the Lunatic Asylum at Montreal, I hereby bind and oblige myself to remove when required by you to do so; to bury ___ in case of death or to pay the funeral expenses; to keep a proper stock of necessaries, and generally to fulfill all the obligations required by the regulations of the Institution. I am, Gentlemen, yours. To the acting Commissioners, Lunatic Asylum, Montreal.»

48 APC, RG 4 B 65, vol. 2, dossier 1846, Worth à J. B. C. Thresler, 24 juillet 1845; RG $4 \mathrm{C} 1$, vol. 8, doc. 1369, les commissaires à Murdoch, 31 mars 1840.

49 Voir APC, RG 4 C 1, vol. 31, doc. 7688, et RG 4 C 1, vol 37, doc. 8557. Ce renseignement apparaît dans une déposition du serviteur en question qui allègue que le surintendant Worth était violent et battait certains des internés lorsqu'il était sous l'influence de l'alcool. (RG 4 C 1, vol. 31, doc. 7688). Bien que ces accusations semblent avoir été fondées, rien ne fut prouvé par l'enquête dirigée par les commissaires (RG 4 C 1, vol. 37, doc. 8857), qui n'avaient d'ailleurs à prouver quoi que ce soit. Par conséquent aucune sanction ne fut imposée et Worth et son épouse conservèrent leur poste jusqu'à la fermeture de l'asile en 1845

50 Rules and Regulations..., 12-13.
} 
mission) fut nommé médecin attitré de l'asile ${ }^{51}$. Le médecin était chargé d'examiner chaque patient à son arrivée, de lui assigner une diète et de lui prescrire divers traitements à mesure qu'il le jugeait nécessaires ${ }^{52}$. En fait, la thérapie en usage correspondait à celle qui se pratiquait à l'époque dans les autres institutions asilaires d'Europe et d'Amérique du Nord. En ce qui a trait aux soins prodigués et au comportement du personnel envers le patient, ils semblent avoir été inspirés des règles générales édictées par Philippe Pinel dans son Traité médicophilosophique ${ }^{53}$. Ainsi, il était expressément recommandé aux membres du personnel - surintendants, matrone, infirmières (gardiens dans la section des hommes) et servantes (serviteurs) - de toujours se comporter face aux internés avec bienveillance et douceur, de les encourager par de bonnes paroles et de ne rien tenter qui puisse les effrayer ou leur faire croire qu'on pouvait se moquer d'eux. Le personnel vivait à l'asile avec les patients et devait veiller à ce que ceux-ci mènent une vie bien réglée: lever à 6:30 heures et coucher à 21:00 heures aux heures d'été (du 1er avril au 30 septembre), et à 7:30 heures et 20:00 heures respectivement aux heures d'hiver (du ler octobre au 31 mars). Le déjeuner était servi à 8:30 heures, le dîner à 11:30 heures et le «thé» à 19:00 heures. La stricte surveillance exercée sur les aliénés ne devait cependant pas empêcher ces derniers de se sentir en parfaite liberté aux endroits qui leur étaient accessibles ${ }^{54}$. Bien entendu, le travail et les activités en plein air étaient à l'honneur. Ainsi, les patients «plus calmes» étaient amenés par groupes de quatre ou cinq dans une cour extérieure aménagée à leur intention. Là, sous la surveillance d'un gardien, ils coupaient du bois ou s'adonnaient à différents jeux ${ }^{55}$. Pour les femmes, il semble probable que les tâches ménagères (lavage, cuisine) aient constitué l'élément prépondérant de leur «thérapie de travail». Les aliénés plus agités, quant à eux, étaient soumis au traitement des bains-douches ${ }^{56}$ : le malade se trouvait enfermé dans une cuve pendant une quinzaine de minutes alors que l'on déversait sur lui de l'eau froide ${ }^{57}$. Pour les patients plus dangereux et violents, on avait prévu des contentions ${ }^{58}$, ou l'isolement dans l'une des chambres ${ }^{59}$.

\footnotetext{
51 APC, RG 4 C 1, vol. 42, doc. 462, les commissaires au secrétaire provincial, 28 avril 1841. Le médecin recevait 75 livres annuellement (cours d'Halifax), ce qui semble démontrer que ses services étaient réguliers. A titre comparatif, mentionnons qu'un gardien recevait 25 livres par année. Tous les autres commissaires semblent quant à eux avoir agi à titre bénévole.

52 Rules and Regulations..., 8-14.

53 P. Pinel, Traité médico-philosophique (Paris, an IX).

54 Rules and Regulations..., 7-10.

55 APC, RG 4 C 1, vol. 50, doc. 1659, les commmissaires au secrétaire provincial, 25 août 1841, et ibid, doc. 1653, J. Boston au secrétaire provincial, 24 août 1841. Le qualificatif "plus calme» semble avoir été assez relatif puisqu'à une occasion au moins, un patient profita d'un moment de relâchement dans la surveillance pour décapiter un compagnon avec la hache servant à la coupe du bois!

56 APC, RG 4 C 1, vol. 37, doc. 8857, les commissaires à Murdoch, 20 février 1841

57 APC, RG 4 C 1, vol. 31, doc. 7688, D. Arnoldi au gouverneur général, 29 décembre 1840 .

58 APC, RG 4 C 1, vol. 37, doc. 8857, les commissaires à Murdoch, 20 février 1841.

59 APC, RG 4 B 65, vol. 2, dossier 1845, Worth à J. B. C. Thresler, 24 juillet 1845.
} 
Au cours de ses cinq premières années d'existence, soit de 1839 à 1844, le Montreal Lunatic Asylum admit 196 patients dont 123 reçurent leur congé, 98 étant considérés comme guéris, et les 25 autres, comme améliorés $^{60}$. Bien qu'un tel «taux de guérison» $(60 \%)$ paraisse étonnant, il ne diffère pas de celui qu'on observe ailleurs à la même époque: les asiles de Toronto ou celui de Worcester aux États-Unis affichaient à vrai dire des taux comparables ${ }^{61}$. Ces statistiques ne font du reste que refléter une tendance générale à l'époque, née de l'élan quasi messianique qui avait vu se multiplier les asiles dans les années 1815-1830 en Europe et 1830-1840 en Amérique du Nord. En effet, comme on ne connaissait alors aucune médication ou traitement psychothérapeutique vraiment efficace, on avait misé énormément sur l'asile dans la guérison des maladies mentales. Le choix du terrain, on l'a vu, et l'architecture de l'édifice étaient devenus une véritable science dans le traitement des troubles mentaux. Il est certain qu'au début, l'érection d'institutions assez vastes, pour ne pas être surpeuplées et comprenant des salles communes, des ateliers et des terrains récréatifs, constituait une amélioration dans la condition des patients. Toutefois, ces conditions nouvelles ne justifiaient certainement pas des «taux de guérison» aussi élevés que ceux que nous avons évoqués. En fait, les médecins et directeurs d'asiles, qui s'étaient faits les promoteurs intéressés d'institutions de ce genre, avaient surestimé les propriétés curatives de l'asile et ils eurent facilement tendance à déclarer guéris ou améliorés bon nombre de patients qui, bien souvent, n'étaient atteints que de troubles mineurs ou passagers. Nombreux cependant étaient les malades qui recevaient leur congé sans vraiment être guéris et qui revenaient peu de temps après.

Un problème plus lourd de conséquence encore allait toutefois peser de facon déterminante sur l'évolution de l'institution asilaire et effacer le bel optimisme des débuts de l'ère de l'asile: le surpeuplement. En effet, à cause de l'accumulation des cas les plus sérieux, dits «incurables», qui résultait en partie de l'inexistence de moyens de guérison efficace et en partie aussi de la hausse constante des admissions, les asiles, mêmes gigantesques, devinrent rapidement saturés de cas dits incurables, au point où l'admission de cas considérés comme moins graves ou qui offraient certains espoirs de guérison devint impossible. Faute d'espace adéquat et de ressources humaines et financières suffisantes, on cessa de prodiguer le traitement moral aux patients. A partir de ce point, finis les taux de guérison mirobolants: l'asile devint un immense réceptacle où les malades n'étaient plus guéris, mais plutôt

${ }^{60}$ APC, RG 4 B 65, vol. 2, dossier 1843-44, les commissaires au gouverneur général, 21 août 1844 .

61 APC, RG 4 B 65, vol. 2, dossier «Toronto Lunatic Asylum», voir également A. Deutsh, The Mentally Ill in America (New York, Columbia University Press, 1949), 154-157 et 230; L. V. Bell, Treating the Mentally Ill (New York, Preager, 1980), 29. 
contenus et gardés, dans l'espoir que certains d'entre eux guérissent d'eux-mêmes.

L'amorce de ce processus est particulièrement visible dans le cas du Montreal Lunatic Asylum, qui ne tarda pas lui non plus à se voir aux prises avec un problème de surpopulation:

TABLEAU 1

Accroissement de la population au Montreal Lunatic Asylum (1840-1845)

\begin{tabular}{|lccc|}
\hline \multicolumn{1}{|c}{ Date } & Hommes & Femmes & Ensemble \\
\hline 8 octobre 1840 & 7 & 12 & 19 \\
5 avril 1841 & 14 & 13 & 27 \\
8 juillet 1843 & 22 & 26 & 48 \\
24 juillet 1845 & 24 & 23 & 47 \\
28 septembre $1845^{*}$ & $?$ & $?$ & 52 \\
\hline
\end{tabular}

Source: APC, RG 4 C 1, vol. 24, doc. 6632, les commissaires au gouverneur général, 8 octobre 1840; RG 4 C 1, vol. 39, doc. 173, les commissaires au gouverneur général, 5 avril 1841; RG 4 C 1, vol. 83, doc. 1459, W. Lunn au secrétaire provincial, 8 juillet 1843; PG 4 B 65, vol. 2, dossier 1846, Worth à J. B. C. Thresler, 24 juillet 1845; PG 4 B 65, vol. 2, dossier 1846 .

* Date du transfert des malades à l'asile temporaire de Beauport.

Dès 1841 , alors que l'asile ne comptait que 27 patients, les commissaires avaient déjà commencé à se plaindre de la surpopulation:

....in the female side there are 12 patients 2 attendants and the matron with her children: 15 grown persons (excluding the childrens) in a space little more than sufficient for half this mumber, as several of the patients are violent and should (if possible) be confined in seperate sleeping appartments. ${ }^{62}$

A partir de ce moment, le problème n'allait cesser de croître. En 1845, alors que 51 personnes résidaient à l'asile, on pouvait compter trois patients par chambre. Deux étaient installés dans un corridor, un dans un hall où l'on avait aussi installé deux gardiens ${ }^{63}$.

En fait, le processus de surpeuplement s'était amorcé dans les tout premiers temps du Montreal Lunatic Asylum. Dès 1840, en effet, le docteur Arnoldi, le médecin affecté à la prison commune de Montréal et chargé d'y dépister les malades, se plaignait du fait qu'on avait refusé

\footnotetext{
$1841_{63}$

63 APC, RG 4 B 65, vol. 2, dossier 1846, Worth à J. B. C. Thresler, 24 juillet 1845.
}

62 APC, RG 4 C 1, vol. 39, doc. 173, les commissaires au gouverneur général, 5 avril 
ou renvoyé de l'asile plusieurs patients qu'il avait tenté d'y faire admettre $^{64}$. Les commissaires se défendirent ainsi:

....upon opening the present temporary asylum, all persons who then in confinement in the jail, in any of weak or deranged intellect and who have been commited as vagrants were transfered to the asylum, and this was unhesitatingly permitted, as the accomodation was not then required for more urgent cases. Since that period several patients have been received, most of whom are in a state of violent mental excitement. Applications were lately made to receive three women, all violent and unmanageable by their friends; in order to make room for these persons it became necessary to discharge those attended (by) Dr Arnoldi. ${ }^{65}$

Ainsi, la saturation de l'espace par des patients «chroniques» au détriment d'autres offrant quelqu'espoir de guérison, ce mal dont furent atteints la grande majorité des asiles occidentaux du 19e siècle, s'était rapidement répandu au Montreal Lunatic Asylum. Le président de l'institution était bien conscient du problème et ne se faisait d'ailleurs, en 1844, plus beaucoup d'illusions à ce propos:

The crowding of insane patients together as is absolutely necessary in the present limited space, can afford little hope of advantage, further than their safe custody, and besides, the occupation of this very abridged accomodation, for want of this class, necessarily excludes any more encouraging cases, and still further, limit the utility of the present temporary asylum. ${ }^{66}$

Même si le surpeuplement taxait lourdement la qualité de ses soins, il semble cependant que le Montreal Lunatic Asylum ne resta pas ouvert assez longtemps pour qu'ils se détériorent au même point qu'ailleurs. C'est du moins l'impression qu'on a à la lecture d'un éditorial du Montreal Medical Gazette, daté du 1er août 1844:

Where is our lunatic asylum... Go to the commom gaol of the city, and there, in the female department, you will find them huddled together by three in the same cell, and at night made to sleep in the narrow passages which separate the miserable cells on either sides. Of the receptacle of Canada West, we hear that there is but one at Toronto and... we are informed that ours is a paradise to it. $^{67}$

64 APC, RG 4 C 1, vol. 4, doc. 821, D. Arnoldi au gouverneur général, 24 février 1840

65 APC, RG 4 C 1, vol. 8, doc. 1369, les commissaires à Murdoch, 31 mars 1840.

66 APC, RG 4, vol. 2, dossier 1844, J. Boston au gouverneur général, 21 août 1844.

67 Montreal Medical Gazette, ler août 1844, 194. Cet éditorial est l'un des rares témoignages publics concernant les conditions de vie à l'asile. La comparaison avec l'asile de Toronto est intéressante puisque les deux institutions ont connu, au début du moins, un développement en plusieurs points similaire. Principalement public (il possédait quelques places payantes pour les gens aisés), l'asile de Toronto était de taille comparable au Montreal Lunatic Asylum, puisqu'il comptait 57 patients au 31 août 1844 (APC, RG 4 B vol.65, vol. 2, dossier «Toronto Lunatic Asylum»). Plusieurs rapports y ont par la suite condamné les conditions faites aux malades (A. Paradis, «L'asile temporaire de Toronto (1841-1850) ou l'impossibilité provisoire de l'utopie asilaire», Santé mentale, 3,1 (juin 1978): 18-36) ce qui peut en partie confirmer l'évaluation du Montreal Medical Gazette. 
Bien qu'imparfaites, il semble toutefois que les conditions de vie des patients du Montreal Lunatic Asylum aient été supérieures à celles que les malades mentaux avaient dû endurer jusque-là dans les prisons et les hôpitaux généraux. Il suffit pour s'en rendre compte de comparer les rapports du Grand Jury de la ville de Québec de 1845 à celui du Grang Jury de Montréal, qui faisait suite à une visite à l'asile local et dans lequel on pouvait lire:

They visited the part of the gaol used as a lunatic Asylum, under the care of Mr Worth who appears to have used his best endeavour towards contributing to the comfort of the persons confined but they cannot avoid regretting that this populous province still remains without any proper place of refuge. ${ }^{68}$

Il est à noter que ce jugement porté par le Grand Jury reste positif à certains égards et ce, malgré le problème aigu de surpopulation au Montreal Lunatic Asylum, avec la présence de 48 patients au moment de leur visite.

La fonction principale de l'asile était d'isoler les individus susceptibles de porter atteinte à la «sécurité» et à la «moralité» de la société et d'en faire à nouveau des citoyens productifs. Il n'est donc pas étonnant que ce soit dans les prisons que le Montreal Lunatic Asylum ait recruté la majorité de ses patients, du moins dans les débuts. Il semble on outre qu'on ait eu tendance à admettre surtout des personnes dans la fleur de l'âge, donc plus susceptibles de constituer un danger pour la société; $87 \%$ des patients ( $86 \%$ à l'asile de Toronto) étaient en effet âgés de 20 à 49 ans ${ }^{69}$.

La répartition des patients de la première institution asilaire bascanadienne suivant l'origine ethnique est par ailleurs fort intéressante à observer. Elle semble corroborer notre hypothèse à l'effet qu'au BasCanada, l'asile soit né de concepteurs britanniques, en réaction à l'accroissement brutal de l'indigence urbaine suscitée par les pressions agraires et démographiques qui agitaient la province dans le deuxième quart du 19e siècle, et par l'immigration massive d'Irlandais miséreux en particulier.

Si l'on tient compte de la proportion des différents groupes ethniques dans la population de Montréal à l'époque, il est frappant de constater à quel point avec $43 \%$ et $22 \%$ des cas respectivement, Irlandais et Écossais sont sur-représentés dans la population asilaire par rapport au maigre $23 \%$ des francophones et au 10\% d'Anglais. En 1844, en effet, la ville de Montréal comptait 42,9\% de francophones, 27,1\%

\footnotetext{
68 APC, RG 4 B 65, vol. 2, dossier 1843-44, le Grand Jury à la Cour des sessions de la paix du district de Montréal, 24 juillet 1843.

APC, RG 4 B 65, dossier 1843-44, les commissaires au gouverneur général, 21 août 1844, et RG 4 B 65, dossier «Toronto Lunatic Asylum».
} 
TABLEAU 2

Origine ethnique des patients au Montreal Lunatic Asylum (1839-1844)

\begin{tabular}{|c|c|c|}
\hline Origine ethnique & nombre & $\%$ \\
\hline Irlandais & 84 & 43,0 \\
\hline Canadiens (français) & 46 & 23,4 \\
\hline Ecossais & 44 & 22,4 \\
\hline Anglais & 20 & 10,2 \\
\hline Autres & 2 & 1,0 \\
\hline Ensemble & 196 & $100 \%$ \\
\hline
\end{tabular}

Source: APC, RG 4 B 65, dossier 1843-44, les commissaires au gouverneur général, 21 août 1844. Ce document contient une liste avec les nom, âge, sexe et origine ethnique de chacune des personnes soignées au Montreal Lunatic Asylum entre 1839 et 1844.

d'Anglais, $21,7 \%$ d'Irlandais et 6,1\% d'Écossais ${ }^{70}$. Les statistiques du Montreal Lunatic Asylum n'ont cependant rien d'étonnant: elles correspondent plus ou moins à la composition ethnique de l'indigence urbaine bas-canadienne à cette époque. Les années 1830-45 furent marquées, ne l'oublions pas, par une arrivée massive d'Irlandais - et, accessoirement, d'Écossais - complètement démunis. Pauvres et miséreux, ceux-ci, souvent sans famille et ne sachant trop où aller, venaient s'entasser dans les villes espérant y trouver quelque secours. Vivant dans la rue, certains aboutissaient à la prison et de là, à l'asile. Les Canadiens français pour leur part, bien qu'ils vécussent au même moment une crise agricole, pouvaient en dernier recours être aidés par quelque membre de leur famille, à la campagne ou ailleurs. Ainsi, risquaient-ils moins d'aboutir à l'asile. Mais, bien plus que la sur-représentation irlandaise ou écossaise, c'est la sous-représentation canadienne-francaise, croyons-nous, qui est responsable d'une telle disproportion ethnique au Montreal Lunatic Asylum.

Comme nous l'avons mentionné, l'asile fit son apparition plus tôt dans les sociétés industrialisées comme la Grande-Bretagne que dans les sociétés à prédominance rurale et traditionnelle comme l'était alors celle du Bas-Canada. Vivant dans des taudis avec un salaire tout juste suffisant pour survivre, une bonne partie de la population britannique s'était habituée, par obligation, à envoyer ses malades dans ces institutions. Ainsi ne parut-il pas anormal aux nouveaux arrivants de placer leurs malades mentaux à l'asile alors que pour les Canadiens français, le Montreal Lunatic Asylum, qui n'était pas né de leurs besoins à eux, ne pouvait représenter autre chose qu'une institution étrangère trans-

70 P.-A. Linteau, «La montée du cosmopolitisme montréalais», Questions de culture, 2 (1982): 23-53. 
plantée dans leur milieu, imposée par la métropole à sa colonie. Administré à l'extérieur comme à l'intérieur par des anglophones, sa construction avait été désirée par la bourgeoisie d'origine britannique et ordonnée par Londres. Rien d'étonnant donc à ce que l'asile ait été rempli de patients originaires des Iles britanniques. Fortement rurale, la société canadienne-francaise n'était tout simplement pas prête encore à accepter aussi facilement d'envoyer ses malades à l'asile. La ferme offrait presque toujours une place pour la garde d'un parent âgé «en démence» ou un enfant «imbécile». Encore en 1856, comme le remarquait l'un des directeurs de l'asile de Beauport, les Canadiens francais étaient réticents à envoyer leurs parents à l'asile ${ }^{71}$. En fait ils allaient y être ethniquement sous-représentés jusqu'aux années 1870 environ, alors que le Québec commençait à s'industrialiser et à s'urbaniser. Les patients d'origine anglaise enfin, constituaient le groupe ethnique le plus sousreprésenté à l'asile (10\%) par rapport à la place qu'ils occupaient dans la population montréalaise $(27 \%)$. Ne faudrait-il pas s'en étonner davantage encore, du fait que l'asile de Montréal et plus tard celui de Beauport avaient été réclamés spécifiquement par des Anglo-Canadiens? Bien au contraire, croyons-nous: l'élite économique montréalaise était à l'époque en majeure partie composée de Canadiens d'origine anglaise. Puisque l'asile représentait alors pour les mieux nantis un agent de contrôle social de la pauvreté urbaine, il est normal qu'ils y aient été sous-représentés, cette institution ne leur étant pas destinée. La majeure partie des Anglais et des membres de l'élite en général qui en avaient besoin se faisaient soigner à la maison ou dans les cliniques privées.

\section{c - Le transfert à Beauport}

En septembre 1845, par suite de pressions de pétitionnaires pour l'ouverture d'un asile permanent et après de longues négociations, le Montreal Lunatic Asylum fermait ses portes et transférait ses patients au nouvel asile «temporaire» de Beauport. Cet événement ne marquait en aucun cas une rupture de l'activité asilaire au Bas-Canada; en fait, il s'agissait avant tout d'un changement de site. Cet épisode de l'histoire asilaire ne va pas cependant sans susciter quelques interrogations. On peut en effet se demander ce qui amena le gouvernement à privilégier Beauport pour la construction d'un asile permanent, ou encore s'interroger sur les motifs qui ont amené les autorités à opter pour une forme institutionnelle semi-privée, qui leur avait été proposée par des médecins-hommes d'affaires de la région de Québec. A première vue,

\footnotetext{
71 Canada (Province), Sessional Papers, J. Douglas, «Report to the Commissionners of the Quebec Lunatic Asylum», Appendix 2, 1856. («This reluctance to place insane persons and idiots in an asylum, is also partly attributed to the strong parental feelings, and the close ties of the Habitants...»). Voir aussi APC, RG 4 C 1, vol. 297, doc. 1136, ainsi que Paradis et al., Essais pour une préhistoire..., 105.
} 
tout cela est assez étonnant si l'on considère que le gouvernement avait déjà entrepris des démarches sérieuses (achat d'un terrain, plans d'un édifice) pour la construction d'un asile public et permanent à Montréal dès 1839 . De plus, avec $54,4 \%$ de la population de la province par rapport à $43,1 \%$ pour le district de Québec ${ }^{72}$, le district de Montréal était tout désigné pour l'obtention d'un nouvel asile. En fait, la réponse à ces questions est relativement simple: parce qu'elle semblait moins urgente, la construction d'un asile ne préoccupait plus autant le gouvernement, qui décida de jouer la carte de l'opportunisme économique et de négocier en vue d'une solution moins coûteuse. Certains, comme les docteurs Badgley et Sutherland, proposèrent bien un projet pour le soin des aliénés dans la région de Montréal, mais, comme leur proposition était assortie d'une entente de dix ans avec le gouvernement, elle fut refusée. On opta plutôt pour la solution des docteurs Douglas et Morrin de Québec qui acceptaient la garde des insensés pour une période de trois ans, moyennant versement par l'État d'un montant forfaitaire par tête, par année ${ }^{73}$. Le nouvel asile allait donc être situé dans une ancienne écurie ${ }^{74}$. Ajoutons qu'à travers les tractations qui entourèrent l'érection du premier asile permanent au Bas-Canada, les considérations financières semblent avoir accaparé les «réformateurs» bien plus que le bien-être des futurs patients. Selon nous, il s'agissait-là de préoccupations d'hommes d'affaires sensibles au rapport coûtbénéfice bien plus que de soucis d'humanistes désireux d'alléger le fardeau de la souffrance humaine.

\section{CONCLUSION}

Tenter d'interpréter l'implantation de l'institution asilaire au Québec en commençant par l'ouverture de Beauport en 1845, comme l'ont fait plusieurs, est à notre avis une erreur. En effet, l'année 1845 - tout comme l'anné 1841 pour Toronto - ne correspond pas à grand chose sur le plan socio-économique qui puisse justifier la naissance de l'asile à cette date précise. Les choses prennent beaucoup de cohérence lorsque l'asile semi-privé de Beauport est remis à la place qu'il a vraiment occupée dans l'évolution de la psychiatrie asilaire au Québec soit celle d'extension et de solution de rechange à un asile public qui avait ouvert ses portes à Montréal en 1839, juste après la publication du Rapport Durham.

La naissance de l'asile au Bas-Canada (et au Haut-Canada aussi, semble-t-il) s'inscrit donc dans une série de mesures envisagées par les Britanniques afin d'assainir le climat social qui avait mené aux troubles

\footnotetext{
72 Canada (Province), Bureau de l'enregistrement et de la statistique, Recensement des Canadas pour 1851-52 (Québec, Imprimeur de la Reine, 1853), xviii.

73 APC, RG 4 B, 65, dossier 1846.

74 British North American Journal of Medecine and Physical Science, 5 (nov. 1849): 193.
} 
de 1837-38. Comme elle reconnaissait déjà l'asile comme instrument de contrôle social dans son milieu d'origine, l'élite anglophone ne pouvait en effet que voir d'un bon oeil son implantation en sol canadien, au moment même où les désordres et l'accroissement de l'indigence urbaine y menaçaient sa suprématie. Avec le retour de l'ordre, en 1839, l'établissement d'un asile permanent au Bas-Canada parut moins pressant pour le gouvernement, qui allait se contenter quelques années plus tard d'une solution de rechange, en donnant son aval à l'ouverture de l'asile de Beauport. Entre-temps, pendant ses six années d'existence, le Montreal Lunatic Asylum, avec des moyens réduits, allait remplir le rôle d'asile à part entière, usant des mêmes méthodes thérapeutiques que la plupart des autres institutions asilaires en Occident. De plus, il ne tardera pas à connaître le surpeuplement, phénomène évolutif courant dans ce genre d'institution à l'époque.

L'étude du Montreal Lunatic Asylum démontre en outre que l'asile fut implanté presqu'artificiellement dans la société québécoise par une puissance qui avait connu une évolution socio-économique différente et qui acceptait déjà ce genre d'institution comme une solution thérapeutique au problème de la maladie mentale. L'hésitation des Canadiens français, qui contrairement aux nouveaux arrivants britanniques semblaient méfiants face à l'asile, et leur manque total de leadership dans la mise en place des premières structures asilaires portent à croire que l'asile n'aurait été implanté que beaucoup plus tard en ce milieu, n'eut été des pressions diverses qui en favorisèrent l'implantation au lendemain des Rébellions. Une chose est sûre: ce n'est que trente à quarante ans après l'épisode du Montreal Lunatic Asylum, lors de la montée de l'industrialisation dans leur milieu, que les Canadiens français ont commencé à s'intéresser aux asiles et à y envoyer les leurs proportionnellement à leur nombre dans la population totale. C'est aussi à cette époque qu'ils remirent le contrôle de ces institutions aux communautés religieuses. Cela ne surprend guère, puisque les autorités ecclésiastiques n'avaient cessé de consolider leur emprise sur la société québécoise depuis le lendemain des Rébellions. L'Église québécoise allait d'ailleurs avoir la mainmise sur les soins aux malades mentaux pour près d'un siècle encore, jusqu'à ce que la Révolution tranquille permette à certains représentants de la nouvelle élite de contester son hégémonie et ses méthodes thérapeutiques. Avant que ne soient ébranlées les murs de Saint-Michel-Archange et de Saint-Jean-de-Dieu, cependant, des milliers d'hommes et de femmes allaient être enfermés dans ces forteresses qui caractérisèrent l'ère de l'asile. C'est là cependant un long chapitre d'une histoire qu'il reste à faire, l'histoire d'une ère qui pourtant avait débuté bien modestement au Québec en 1839 avec l'ouverture du Montreal Lunatic Asylum. 